\title{
MARS 1972
}

\section{AN NÉE- N• 639}

\section{revue internationale de la croix-rouge}

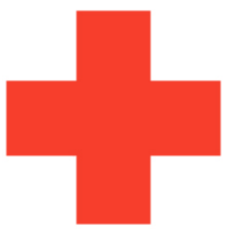

INTER ARMA CARITAS

GENÈVE

COMITÉ INTERNATIONAL DE LA CROIX-ROUGE FONDÉ EN 1863 


\section{COMITÉ INTERNATIONAL DE LA CROIX-ROUGE}

MM. MARCEL, A. NAVILLE, président (membre depuis 1967)

JEAN PICTET, docteur en droit, président de la Commission juridique, vice-président (1967)

HARALD HUBER, docteur en droit, juge féđéral, vice-président (1969)

PAUL RUEGGER, ambassadeur, président du CICR de 1948 à 1955 (1948)

GUILLAUME BORDIER, ingénieur dipl. E.P.F., M.B.A. Harvard, banquier (1955)

HANS BACHMANN, docteur en droit, Stadtrat de Winterthour (1958)

JACQUES FREYMOND, docteur ès lettres, directeur de l'Institut universitaire de hautes études internationales, professeur à l'Université de Genève (1959)

DIETRICH SCHINDLER, docteur en droit, professeur à l'Université de Zurich (1961)

M Me MARJORIE DUVILLARD, infirmière (1961)

MM. MAX PETITPIERRE, docteur en droit, ancien président de la Confédération suisse (1961)

ADOLPHE GRAEDEL, député au Conseil national suisse de 1951 à 1963, ancien secrétaire général de la Fédération internationale des ouvriers sur métaux (1965)

$M$ me DENISE BINDSCHEDLER-ROBERT, docteur en droit, professeur a l'Institut universitaire de hautes études internationales (1967)

MM. JACQUES F. DE ROUGEMONT, docteur en médecine (1967)

ROGER GALLOPIN, docteur en droit, ancien directeur général (1967)

WALDEMAR JUCKER, docteur en droit, secrétaire de l'Union syndicale suisse (1967)

VICTOR H. UMBRICHT, docteur en droit, administrateur (1970)

PIERRE MICHELI (1971)

Membres honoraires: M. JACQUES CHENEVIÈRE, vice-président d'honneur; $\mathrm{M}^{\text {lle LUCIE }}$ ODIER, vice-presidente d'honneur; MM. CARL J. BURCKHARDT, PAUL CARRY, $M^{\text {m}}$ MARGUERITE GAUTIER-VAN BERCHEM, MM. SAMUEL A. GONARD, EDOUARD DE HALLER, PAUL LOGOZ, RODOLFO OLGIATI, FREDERIC SIORDET, ALFREDO VANNOTTI, ADOLF VISCHER.

Direction: MM. JEAN-LOUIS LE FORT, secrétaire général;

RAYMOND COURVOISIER, assistant spécial du président et directeur du département des opérations;

CLAUDE PILLOUD, directeur du département des principes et du droit. 


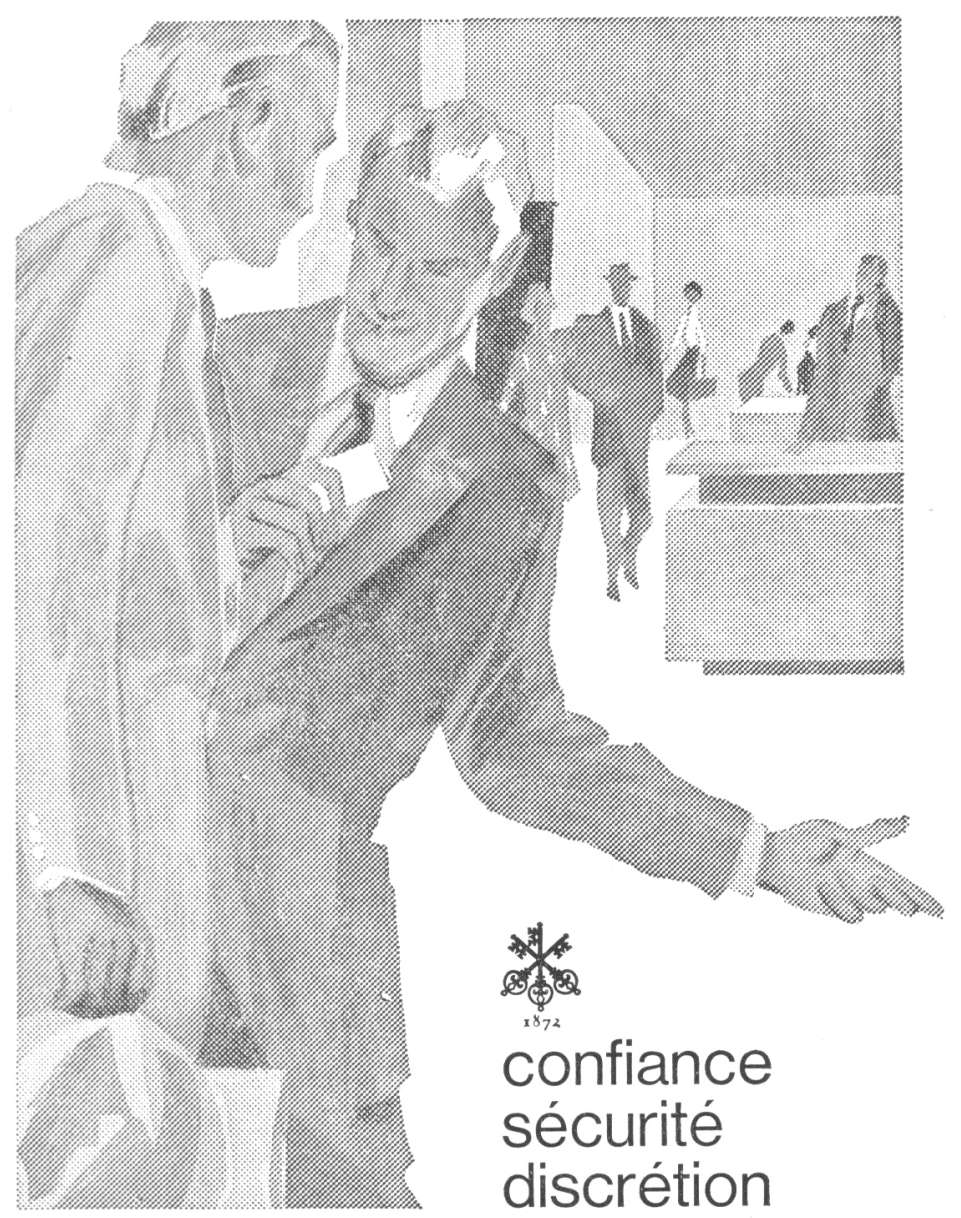

\section{SOCIÉTÉ DE BANQUE SUISSE SWISS BANK CORPORATION}

Siège de Genève: 2, rue de la Confédération Tél. 266211 


\section{SOCIETE FIDUCIAIRE ROMANDE OFOR S.A.}

Place Saint-Gervais 1, Genève - Tél. 317050

- Expertises, revisions et organisations comptables

$\downarrow$ Domiciliation et administration de sociétés

$\downarrow$ Assainissements et liquidations

^ Interventions et conseils en matière fiscale

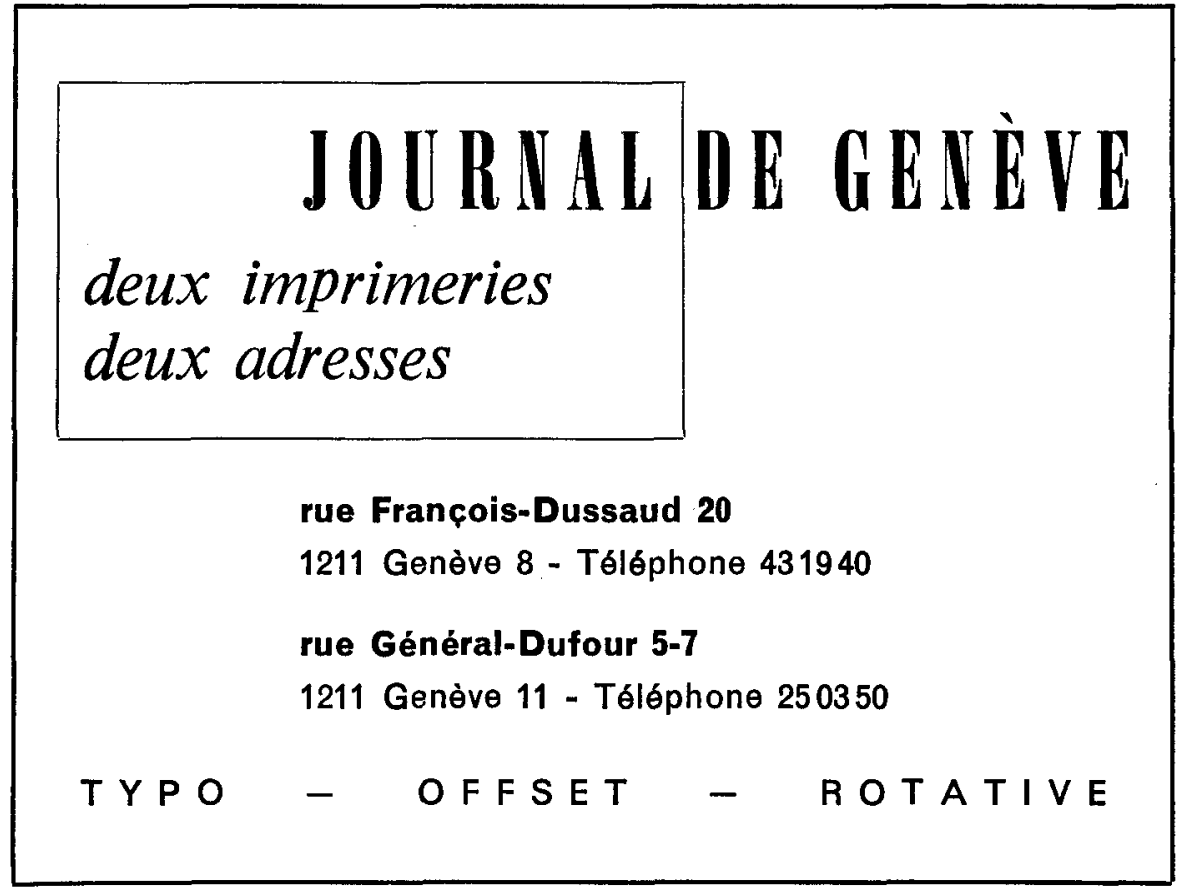




\section{REVUE INTERNATIONALE DE LA CROIX-ROUGE}

MARS $1972-N^{\circ} 639$

\section{SOMMAIRE}

COMITE INTERNATIONAL DE LA CROIX-ROUGE

\section{DANS LE MONDE DE LA CROIX-ROUGE}

FAITS ET DOCUMENTS

LIVRES ET REVUES
Michel Veuthey: Instructions militaires sur le traitement des prisonniers dans la guérilla . . . 139

En faveur des victimes du conflit indo-pakistanais 152 Activités extérieures:

Proche-Orient - République khmère — Laos

République du Vietnam - Bolivie . . . 160

A Genève:

En faveur des victimes d'expériences pseudomédicales. . . . . . . . . . . . . . 164

Développement du Droit international humanitaire - Conférence d'experts de la Croix-Rouge 165

La radiodiffusion au service de la Croix-Rouge 171 «Le Croissant-Rouge et mon pays» dans les pays arabes . . . . . . . . . . . . . 173

Journée mondiale de la Croix-Rouge 1972 . . . 175 France ............... 177

Le problème de la paix . . . . . . . . . . 179 187 
INTERNATIONAL

REVIEW

OF THE RED CROSS

\section{SUPPLEMENTS}

de la ReVue

EN LANGUE ESPAGNOLE

EN LANGUE ALLEMANDE

REVUE INTERNATIONALE DE LA CROIX-ROUGE
Une édition en langue anglaise paraît chaque mois. Elle est en principe identique à l'édition française, et peut être obtenue aux mêmes conditions.

Enseñanza del derecho internacional humanitario Día Mundial de la Cruz Roja 1972 - Primera reunión técnica de los Servicios de información de las Sociedades de la Cruz Roja de América Latina - Panamá.

H. Beer: Die Solidarität des Roten Kreuzes (II) Bibliographie.

Elle est publiée chaque mois par le Comité international de la Croix-Rouge.

7, avenue de la Paix, 1211 Genève 1 (Suisse) - Compte de chèques postaux 12-1767.

Abonnement un an: Fr. 30,--; le numéro: Fr. 3,-.

RÉDACTION : J.-G. LOSSIER

Seuls les textes signés par le Comité international de la Croix-Rouge engagent la responsabilité de celui-ci. 